\title{
Increased extrasynaptic GluN2B expression is involved in cognitive impairment after isoflurane anesthesia
}

\author{
LUNXU LI $^{1}$, ZHENGQIAN LI ${ }^{1}$, YIYUN CAO ${ }^{1}$, DONGSHENG FAN ${ }^{2}$, \\ DEHUA CHUI $^{3}$ and XIANGYANG GUO ${ }^{1}$ \\ ${ }^{1}$ Departments of Anesthesiology and ${ }^{2}$ Neurology, Peking University Third Hospital, Beijing 100191; \\ ${ }^{3}$ Neuroscience Research Institute and Department of Neurobiology, Key Laboratory for Neuroscience, \\ Ministry of Education and Ministry of Public Health, Peking University Health Science Center, \\ Beijing 100083, P.R. China
}

Received February 10, 2016; Accepted April 28, 2016

DOI: $10.3892 /$ etm.2016.3306

\begin{abstract}
There is increasing concern regarding the postoperative cognitive dysfunction (POCD) in the aging population, and general anesthetics are believed to be involved. Isoflurane exposure induced increased N-methyl-D-aspartic acid receptor (NMDAR) GluN2B subunit expression following anesthesia, which was accompanied by alteration of the cognitive function. However, whether isoflurane affects this expression in different subcellular compartments, and is involved in the development of POCD remains to be elucidated. The aims of the study were to investigate the effects of isoflurane on the expression of the synaptic and extrasynaptic NMDAR subunits, GluN2A and GluN2B, as well as the associated alteration of cognitive function in aged rats. The GluN2B antagonist, Ro25-6981, was given to rats exposed to isoflurane to determine the role of GluN2B in the isoflurane-induced alteration of cognitive function. The results showed that spatial learning and memory tested in the Morris water maze (MWM) was impaired at least 7 days after isoflurane exposure, and was returned to control levels 30 days thereafter. Ro25-6981 treatment can alleviate this impairment. Extrasynaptic GluN2B protein expression, but not synaptic GluN2B or GluN2A, increased significantly after
\end{abstract}

Correspondence to: Professor Xiangyang Guo, Department of Anesthesiology, Peking University Third Hospital, 49 North Garden Street, Haidan, Beijing 100191, P.R. China

E-mail:puthmzk@163.com

Professor Dehua Chui, Neuroscience Research Institute and Department of Neurobiology, Key Laboratory for Neuroscience, Ministry of Education and Ministry of Public Health, Peking University Health Science Center, 38 Xueyuan Road, Haidan, Beijing 100083, P.R. China

E-mail: dchui@bjmu.edu.cn

Key words: isoflurane, N-methyl-D-aspartic acid receptor, extrasynaptic GluN2B, cognitive impairment isoflurane exposure compared to non-isoflurane exposure, and returned to control levels approximately 30 days thereafter. The results of the present study indicated that isoflurane induced the prolonged upregulation of extrasynaptic GluN2B expression after anesthesia and is involved in reversible cognitive impairment.

\section{Introduction}

Postoperative cognitive dysfunction (POCD) is generally considered a subtle decline in the cognitive function that occurs in patients following surgery, for which the elderly population is at high risk. While it is believed that general anesthetics produce reversible restraining effects on the brain, it has been shown that use of anesthetics results in prolonged anesthesia-related neuropathophysiological effects after their elimination, such as neuroinflammation (1), blood-brain barrier disruption $(2)$, neurotoxicity $(3,4)$ or persistent depression of synaptic function (5), and are likely involved in the pathogenesis of POCD.

N-methyl-D-aspartic acid receptors (NMDARs) are a major type of excitatory neurotransmitter-gated ionotropic channels with a particularly important role in synaptic plasticity, learning and memory $(6,7)$. NMDARs are one of the important targets of general anesthetics. Previous evidence suggested that isoflurane exposure induced a prolonged NMDAR subunit, and GluN2B upregulation after anesthetic elimination, which was accompanied by changes in cognitive function, albeit the behavioral outcomes were inconsistent (8-12). Functional NMDARs are composed of two obligatory GluN1 subunits, together with two GluN2 subunits or a combination of GluN2 and GluN3 subunits, and most central NMDARs are GluN1/GluN2 assemblies. GluN2A and GluN2B are the primary GluN2 subunits in the forebrain including the hippocampus. After maturation, most GluN2A-containing NMDA receptors are incorporated into synapses. GluN2B-containing NMDA receptors are also present in synapses, but are mainly found extrasynaptically. NMDARs with different subunits and localization trigger different signaling pathways (13), which play different roles in synaptic plasticity, learning and memory, cell survival, excitotoxicity and cell death $(6,14,15)$. 
It is not clear whether synaptic and extrasynaptic GluN2B subunits have different roles in anesthetic-induced cognitive dysfunction. Therefore, the aim of the study was to determine the effects of isoflurane on the expression of the NMDAR subunits, GluN2A and GluN2B, as well as the associated alteration of cognitive function in aged rats. The specific GluN2B antagonist, Ro25-6981, was administered to test the role of GluN2B in anesthetic-induced changes of spatial learning and memory.

\section{Materials and methods}

Animals. Sprague-Dawley rats (male, 20 months of age and weighing 500-600 g) obtained from the Dongchuang Laboratory Animal Center, Changsha (Hunan, China) were used for the experiments. The animals were housed in a temperature- and humidity-controlled room $\left(21 \pm 20^{\circ} \mathrm{C}, 60 \%\right)$ using a 12-h light/dark cycle (light on at 06:00) with food and water available ad libitum. The animals were given an interval of at least 14 days to adapt to their new environment prior to experiments. The animal protocol was approved by the Peking University Biomedical Ethics Committee, Experimental Animal Ethics Branch (approval no. LA 2012-38).

One week prior to anesthesia exposure, the animals were trained to swim in the Morris water maze (MWM) without a platform, four times per day for at least 3 days, and there was a 1 min interval between each 2 min of swimming. Animals with continuous thigmotactic behavior or those floating without swimming for the last 2 days were excluded from experiments.

Isoflurane exposure. The animals were randomly exposed to isoflurane (Baxter Healthcare of Puerto Rico, Guayama, Puerto Rico). Isoflurane (1.5\%) with $2 \mathrm{l} / \mathrm{min} 100 \%$ oxygen as carrying gas $(n=30)$ or vehicle gas (Beijing Millennium City Gas Sales Center (Beijing, China) was employed at 2 1/min $100 \%$ oxygen $(n=30)$ for $4 \mathrm{~h}$. To examine the role of GluN2B in anesthetic-induced changes of spatial learning and memory, an additional two groups ( $\mathrm{n}=30$, respectively) were administered the specific GluN2B antagonist, Ro25-6981 (3 mg/kg, $2 \mathrm{ml} / \mathrm{kg}$, i.p.; Sigma-Aldrich, St. Louis, MO, USA) daily for 6 days, $24 \mathrm{~h}$ after exposure to isoflurane or vehicle gas.

The animals were maintained in an anesthesia chamber during isoflurane or vehicle gas exposure. At the outlet of the chamber, the concentrations of isoflurane, oxygen, and carbon dioxide in the chamber were continuously analyzed with a gas monitor (Datex-Ohmeda, Inc., Louisville, CO, USA). In a previous study, it was shown that this anesthesia protocol did not cause significant changes in blood gas or glucose $(1,2)$.

$M W M$. After $24 \mathrm{~h}$ of treatment, 10 animals from each group were trained for MWM tests for 6 days. Another 10 animals from each group were used for the same tests after 30 days of treatment. The MWM tests were conducted by investigators blind to the group conditions as previously described $(1,2)$. The swimming route was observed by video (Beijing Sunny Instruments Co. Ltd., Beijing, China). The time spent to locate the submerged platform by the animals (defined by the latency cut-off time point of $120 \mathrm{sec}$ ) and the swimming velocity were recorded. On day 6 , a probe trial was performed without the platform. The percentage of time spent in the previous platform quadrant in a 120 -sec period was determined.

Hippocampal tissue harvest and separation of synaptic and extrasynaptic membranes. Following anesthesia exposure, 3 animals from the isoflurane and vehicle gas exposure groups were sacrificed by decapitation at 1, 7 and 30 days post-treatment for harvesting of the hippocampus without MWM tests. The separation of synaptic and extrasynaptic fractions was performed as described by Zhang et al and Goebel-Goody et al $(16,17)$, which was based on the principle that the postsynaptic density (PSD) protein-associated or synaptic fraction was insoluble in Triton X-100, whereas the non-PSD protein-associated or extrasynaptic fraction was soluble in Triton X-100.

Antibodies and immunoblotting. The protein extracted from the hippocampal tissue homogenate, including the total protein, PSD protein-associated (or synaptic) fraction and the non-PSD protein-associated (or extrasynaptic) fraction were used for western blot analysis to test the expression of GluN2A and GluN2B. Total protein $(60 \mu \mathrm{g})$, as well as $40 \mu \mathrm{g}$ protein from synaptic fraction and extrasynaptic fraction per lane were separated electrophoretically in $8 \%$ SDS-PAGE gels, and transferred to nitrocellulose membranes (Millipore, Newyork, USA). The membranes were incubated in rat polyclonal antibodies against GluN2B (1:1,000, cat no.: ab65783) and polyclonal antibodies against GluN2A (1:1,000, cat no.: ab14596) (both from Abcam, Cambridge, MA, USA). The binding of the primary antibodies was detected by fluorescently-labeled secondary antibody (1:10,000), and was visualized by scan-ning membranes in an Odyssey infrared imaging system (both from LI-COR Biosciences, Lincoln, NE, USA). For densito-metric analysis, the signal intensity was quantified as a ratio of GluN2A or 2B/actin and normalized to the values of the corresponding control animals.

Statistical analysis. Statistical analyses were performed using SPSS 20.0 for Windows (SPSS, Inc., Chicago, IL, USA). The values of latency and swimming speed in the MWM test were analyzed using two-way repeated-measures analysis of variance (ANOVA), with Bonferroni post-hoc analysis. The percentage of time spent in the previous platform quadrant and data from the western blot analysis were compared between isoflurane and control groups by one-way ANOVA using Bonferroni post-hoc analysis. Data were presented as mean \pm SEM and statistical significance was set at $\mathrm{P}<0.05$.

\section{Results}

Isoflurane exposure induces reversible spatial learning and memory impairment. We used the MWM test to investigate whether isoflurane affects spatial learning and memory. Fig. 1 shows the results of these experiments in the first week (Fig. 1A-C) and 30 days (Fig. 1D-F) after anesthesia. During the first week after treatment, the time required to locate the platform (latency) in the spatial acquisition training was significantly affected by isoflurane treatment compared to the control group (Fig. 1A; $\mathrm{P}<0.01$ ). A probe trial was conducted to evaluate reference memory at the end of learning. The time 
A

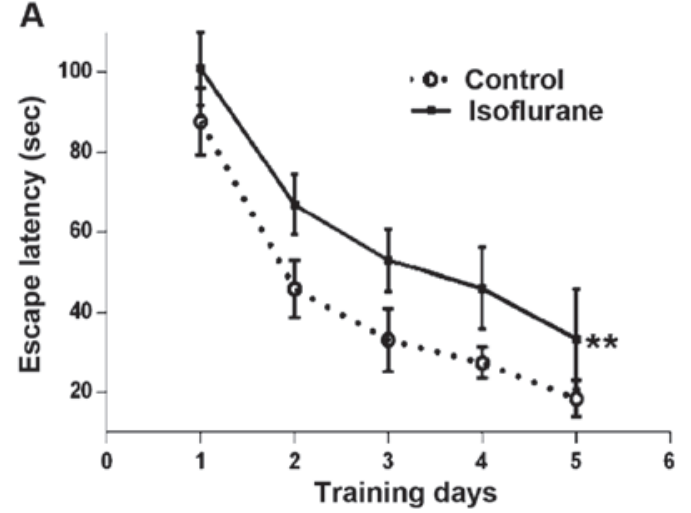

B

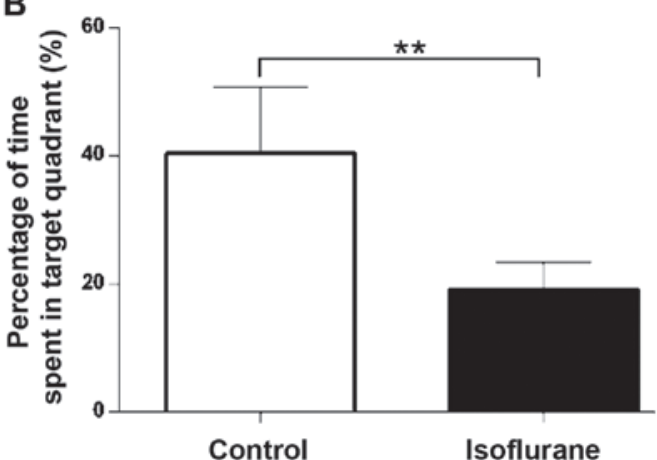

C

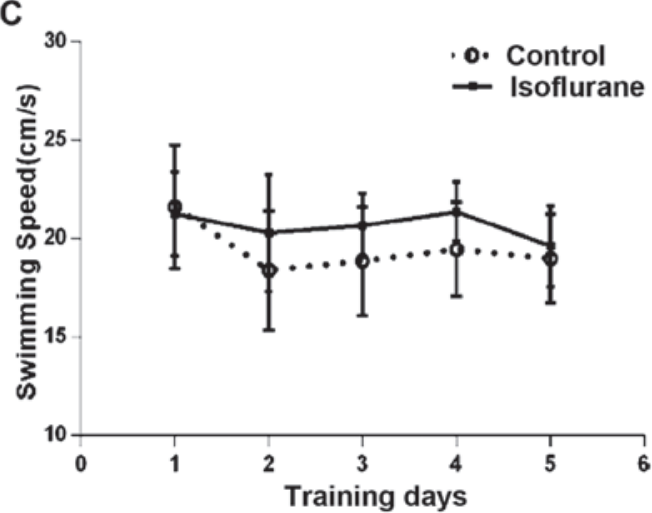

D

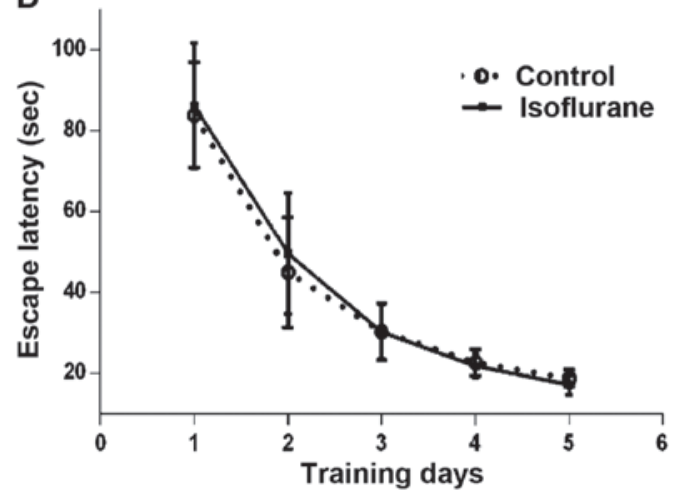

E

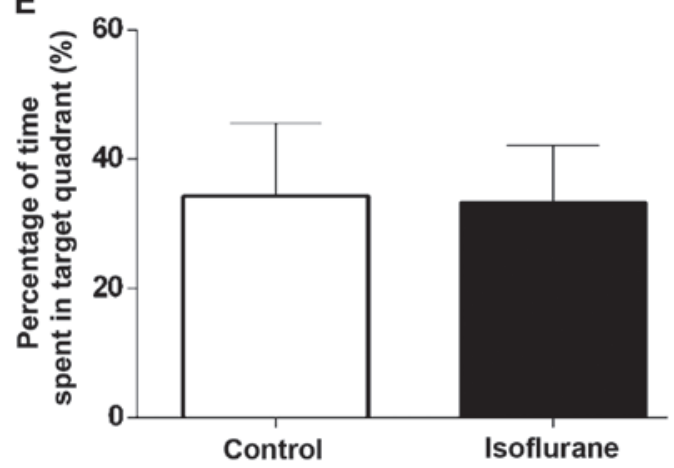

$\mathbf{F}$

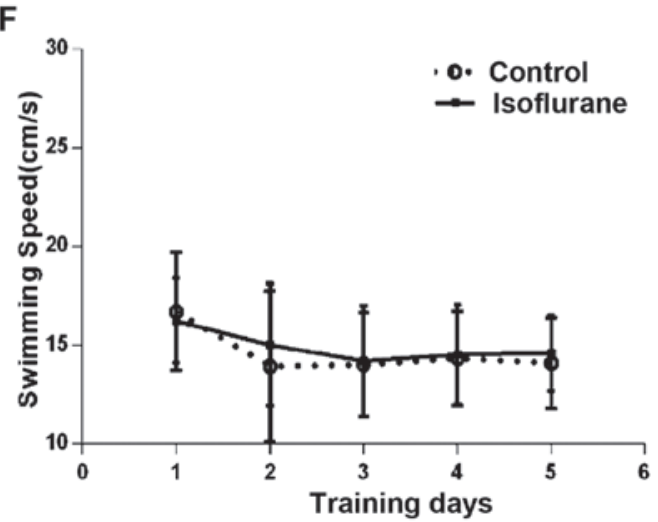

Figure 1. Effect of isoflurane on the spatial memory function in the MWM task during the (A-C) first week and (D-F) 30 days after treatment. (A and D) Escape latency, $(\mathrm{B}$ and $\mathrm{E})$ percentage of time spent in target quadrant $(\%)$, and $(\mathrm{C}$ and $\mathrm{F})$ swimming speed. Results are shown as means $\pm \mathrm{SEM}(\mathrm{n}=10)$. ${ }^{* *} \mathrm{P}<0.01$. MWM, Morris water maze.

spent in the platform area by the rats in the isoflurane group was shorter than that in the control group (Fig. 1B; $\mathrm{P}<0.01$ ). Isoflurane treatment had no effect on swimming speed compared to the control group (Fig. 1C; P>0.05). Rats from each group not receiving MWM test during the first week post-anesthesia underwent the same procedures of MWM test 30 days after the treatment. We found that there were no differences in escape latency and percentage of time spent in the target quadrant between the isoflurane and control groups (Fig. 1D and E; P>0.05). The swimming speed between the two groups showed no difference during the first week and 30 days after treatment (Fig. 1F; P>0.05). These results indicated that the spatial learning and memory in MWM test was impaired at least 1 week following isoflurane exposure, but recovered to the control level 30 days after the treatment.
GluN2 subunit expression in the hippocampus increases following isoflurane exposure. Rats in the isoflurane and control groups were decapitated at 1,7 and 30 days posttreatment to collect the hippocampus without MWM tests. The hippocampal NMDARs GluN2A and GluN2B subunit protein expression is shown in Fig. 2. The synaptic GluN2A levels showed no difference between isoflurane and control groups at 1, 7 and 30 days following treatment (Fig. 2A-C; $\mathrm{P}>0.05$, between isoflurane and control groups). The levels of total GluN2B protein in the hippocampus increased $24 \mathrm{~h}$ after the isoflurane exposure compared with the control group (Fig. 2D; $\mathrm{P}<0.05$, between the isoflurane and control groups). At 7 days after treatment, the GluN2B levels in the isoflurane group were higher than those in the control group although there was no statistical significance (Fig. 2D; 

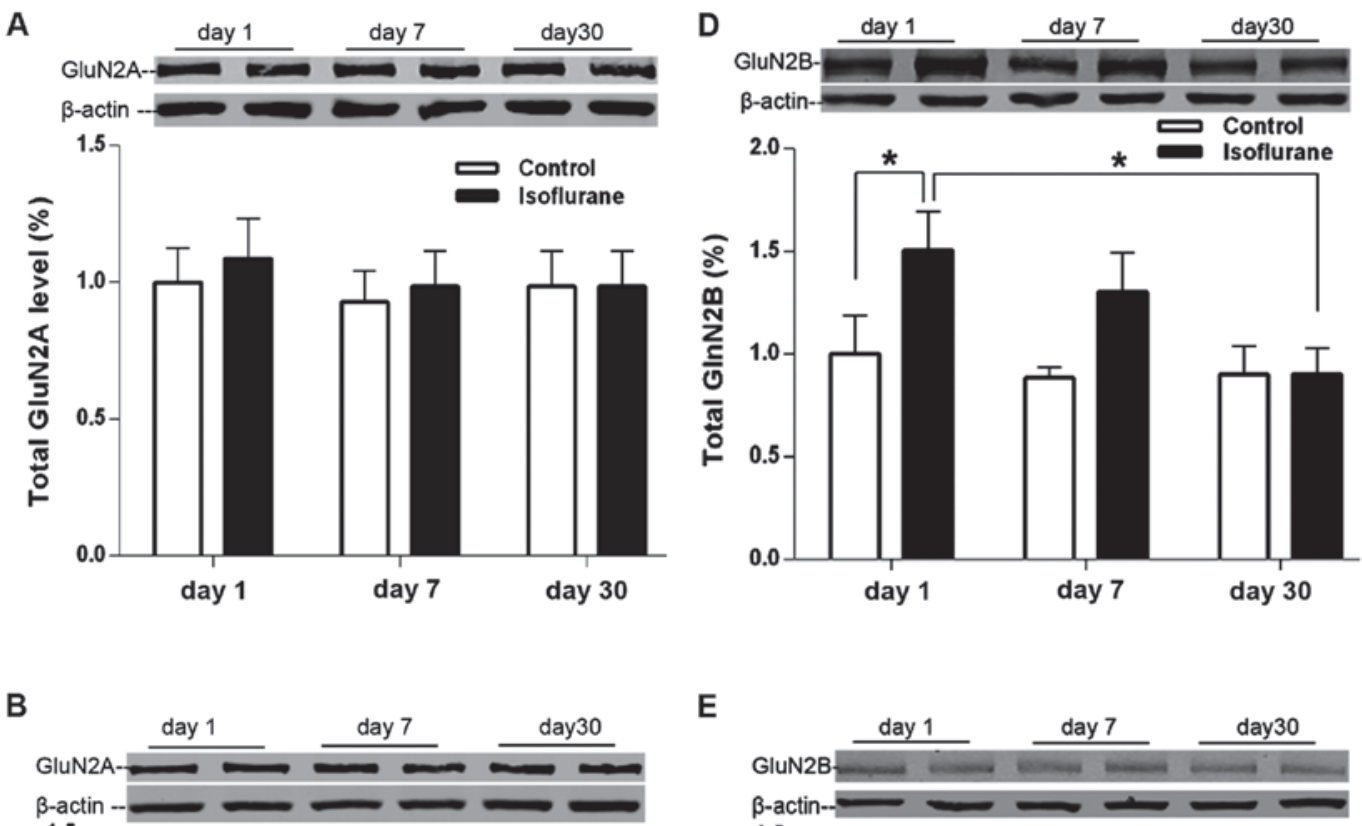

E
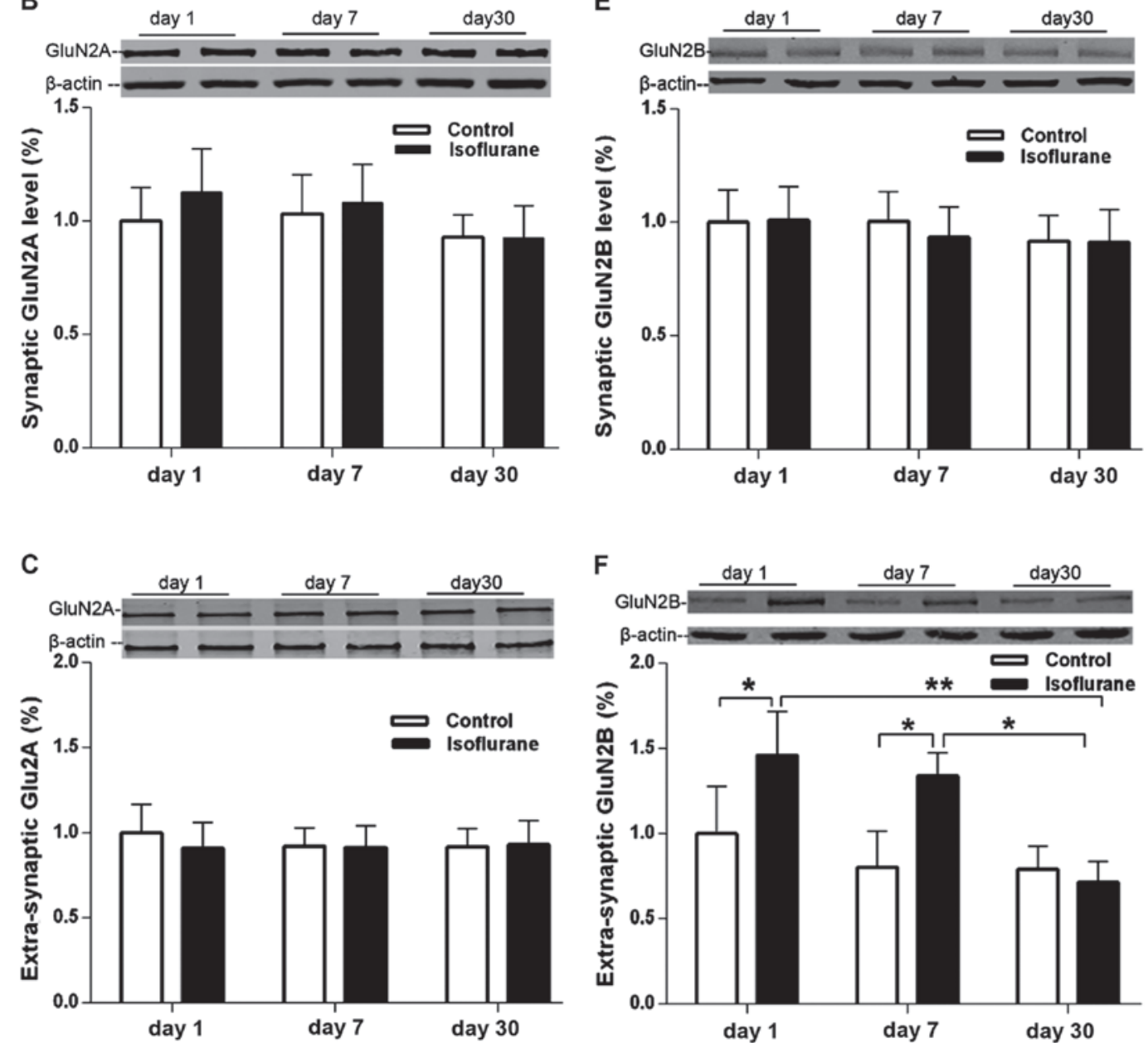

Figure 2. Hippocampal NMDARs GluN2A and GluN2B subunit protein expression. Immunoblots against (A-C) GluN2A, (D-F) GluN2B in isoflurane- and vehicle-treated rats at 1,7 and 30 days post-treatment. ${ }^{*} \mathrm{P}<0.05,{ }^{* * *} \mathrm{P}<0.01$. Total Glu2B subunit and extrasynaptic GluN2B were upregulated at 1 and 7 days after isoflurane treatment, and returned to the control levels at 30 days after treatment, whereas the Glu2A and intrasynaptic GluN2B protein levels show no differences between the isoflurane and control groups.

$\mathrm{P}>0.05$ ), and they returned to the control levels after 30 days following the isoflurane exposure (Fig. 2D; P $>0.05$ between the isoflurane and control groups). The levels of extrasynaptic GluN2B in the hippocampus also increased $24 \mathrm{~h}$ following isoflurane exposure compared with the control group (Fig. 2F; $\mathrm{P}<0.05)$, and remained higher than that in the control group at 7 days after treatment (Fig. $2 \mathrm{~F}, \mathrm{p}<0.05$ ). However, the levels also returned to the control levels 30 days after the treatment (Fig. 2F, P>0.05 between the isoflurane and control groups). The synaptic levels of GluN2B showed no difference between the isoflurane and control groups at 1, 7 or 30 days after treatment (Fig. 2E, $\mathrm{P}>0.05$ ). 
A

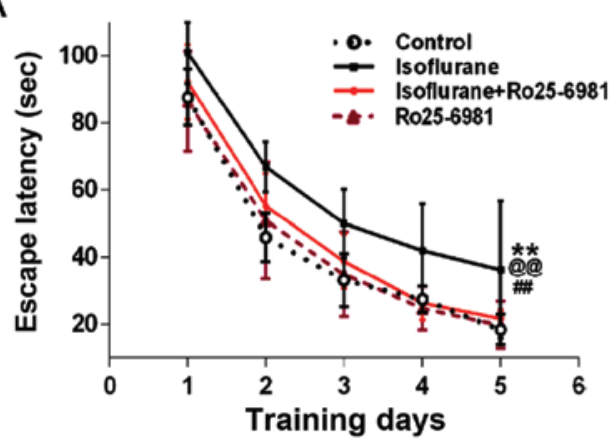

B

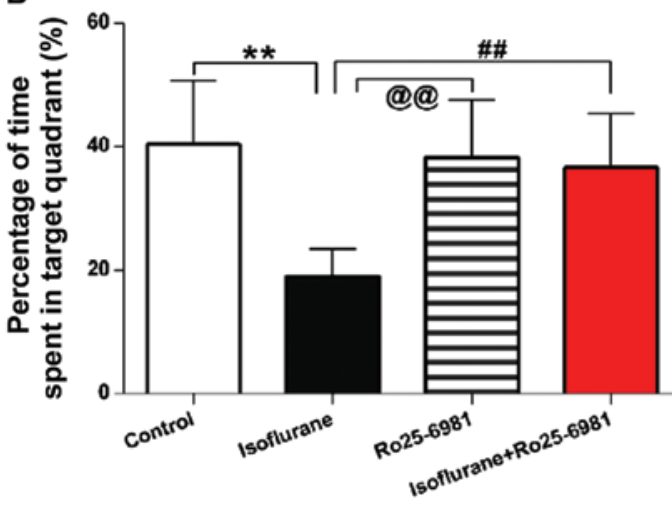

C

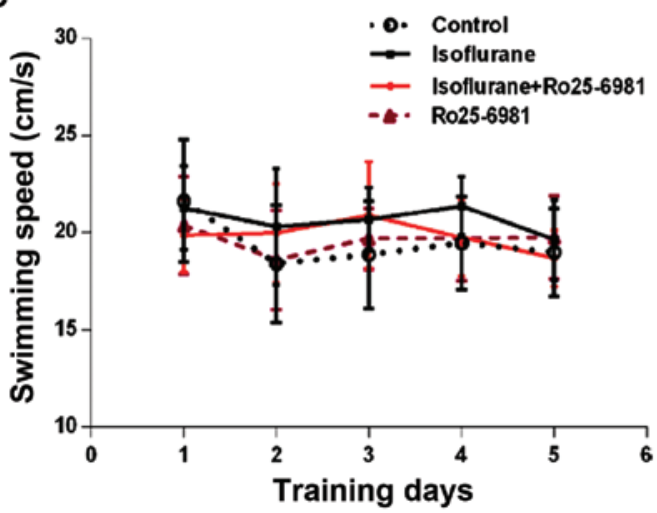

D

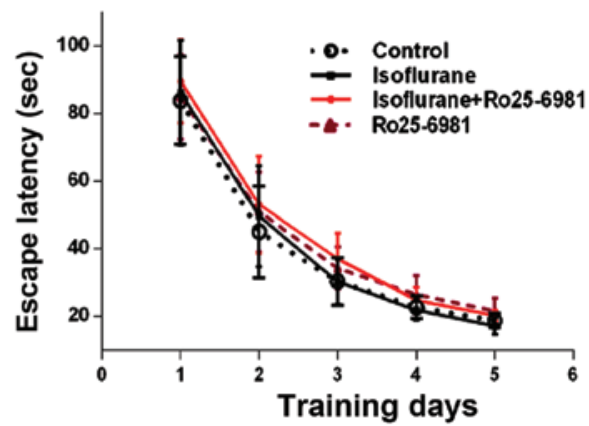

E

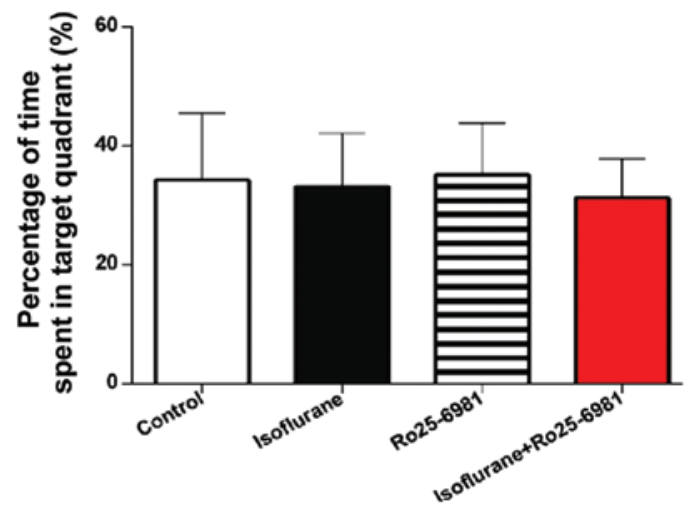

$\mathbf{F}$

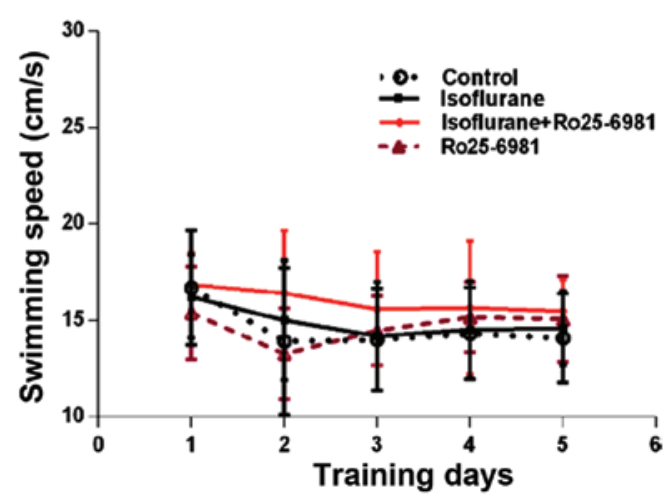

Figure 3. Ro25-6981 alleviated isoflurane-induced impairment of spatial memory function in the MWM task during the first week (A-C) and 30 days (D-F) after treatment. Escape latency (A, p $<0.01 ; \mathrm{D}, \mathrm{p}>0.05)$; Percentage of time spent in target quadrant (\%) (B, p $<0.01 ; \mathrm{E}, \mathrm{p}>0.05)$; Swimming speed $(\mathrm{C}$ and $\mathrm{F}, \mathrm{p}>0.05)$. Results are presented as means $\pm \mathrm{SEM}(\mathrm{n}=10) .{ }^{* *} \mathrm{p}<0.01$, between isoflurane group vs. control group; ${ }^{\circledR} \mathrm{p}<0.01$ between isoflurane group vs. isoflurane + Ro25-6981 group; ${ }^{\# \#} \mathrm{p}<0.01$, between isoflurane vs. Ro25-6981 group. MWM, Morris water maze.

GluN2B-specific antagonist Ro25-6981 alleviates spatial learning and memory impairment induced by isoflurane exposure. During the first week after treatment, Ro25-6981 significantly reduced the latency of rats exposed to isoflurane in spatial acquisition training (Fig. 3A; $\mathrm{P}<0.05$ between the isoflurane and isoflurane + Ro25-6981 groups), and the time spent in the platform area in the probe trial was prolonged by Ro25-6981, compared to the rats treated only with isoflurane (Fig. 3B, $\mathrm{P}<0.01$ between the isoflurane and isoflurane + Ro25-6981 groups). However, there were no differences in the two aforementioned outcomes in the four groups 30 days after treatment (Fig. 3D and E; P>0.05). No differences in swimming speed in the four groups were identified $(\mathrm{P}>0.05)$. No differences in swimming speed in the four groups were identified (Fig. $3 \mathrm{C}$ and $\mathrm{F} ; \mathrm{P}>0.05$ ).
The latency during the acquisition training was not affected by Ro25-6981 alone, when compared with the control levels during the first week and 30 days thereafter (Fig. 3A and D; $\mathrm{P}>0.05$ ). The time spent in the platform area in the probe trial was not different between the control and Ro25-6981 groups during the 7 or 30 days after treatment (Fig. 3B and E; P>0.05).

\section{Discussion}

POCD has become a more common phenomenon in the aging population, albeit its mechanism remains unclear. Anesthetics may partially consitute the underlying problem. The majority of investigations pertaining to anesthetic-induced cognitive dysfunction were focused on neurotoxicity and cell death, and the majority of supporting evidence stemmed from 
A

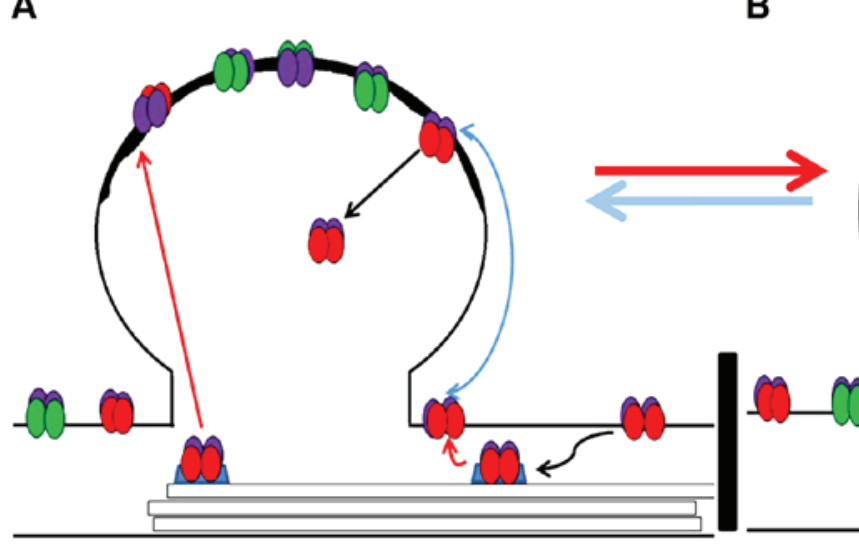

GluN1

GluN2A

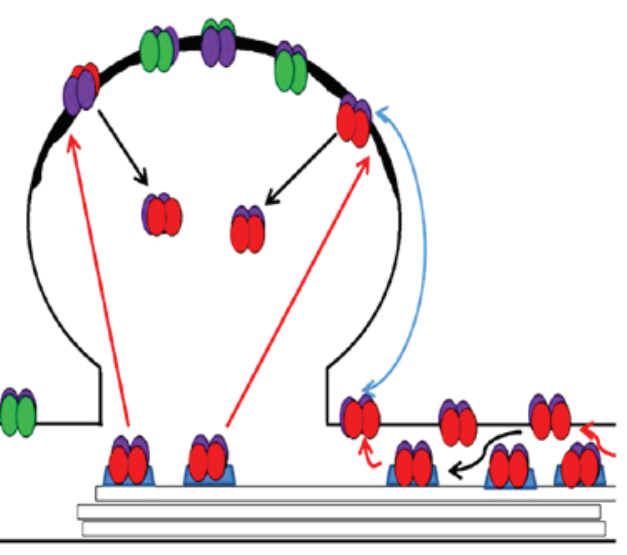

GluN2B

Figure 4. Isoflurane-induced upregulation of extrasynaptic GluN2B. Isoflurane-induced expression of (A and B) GluN2B pre- and post-isoflurane exposure, respectively, combined with curtailing the synaptic GluN2B location in aged animals by modification, such as dephosphorylation with CDK5, may result in the upregulation of extrasynaptic GluN2B without changes in synaptic GluN2B levels and lead to inhibition of the synaptic function.

cultured cells and the developing brain $(18,19)$. Although there are clinical studies that support that age is a risk factor for cognitive dysfunction, there is little evidence to show that the aforementioned neurotoxicity and cell death are involved in cognitive dysfunction in animal models on aging $(3,20)$. On the other hand, epidemiological evidence has demonstrated that the prevalence rate of POCD has decreased over time, suggesting that POCD in most patients may be temporary and self-limiting (21-23). Stratmann et al identified that aged rats exposed to isoflurane showed no evidence of ongoing cell death, impaired hippocampal neurogenesis, or longterm cognitive impairment (24). Cognitive impairment by anesthetics in the aging brain manifest certain temporary characteristics of Alzheimer's disease, such as elevated $A \beta$ production and $\tau$ hyperphosphorylation (25), which caused synaptic dysfunction without obvious neural loss at the early phase of this disease (26-30). Evidence suggests that inhibitory ionotropic receptors, $\alpha-5$ subunit containing $\gamma$ aminobutyric acid-A receptors ( $\alpha-5$ GABAARs) can be upregulated and maintain a persistent inhibitory current for 1 week even after a single dosage of etomidate (5). Given the aforementioned evidence, it is reasonable to hypothesize that delayed inhibitory effects in neural activity secondary to anesthetic exposure, even after their elimination, may partially account for cognitive dysfunction, in addition to cell death.

NMDARs are a target of general anesthetics, and play an important role in synaptic plasticity, learning and memory (31). Previous findings have shown that the effects on NMDARs by isoflurane can be sustained beyond the elimination of anesthetics and are involved in changes in cognitive function, albeit the behavioral outcomes were inconsistent (8-12). Our results demonstrate spatial learning and memory in aged rats tested in the MWM. These rats showed impairment of these parameters for at least 1 week following isoflurane exposure, and returned to the control levels 30 days later, suggesting that isoflurane did not induce permanent damage to the central nervous system. We also found that isoflurane exposure for $4 \mathrm{~h}$ may induce prolonged changes in GluN2B expression levels for at least 1 week following isoflurane treatment. It is a common pharmacological phenomenon that chronic treatment with receptor antagonists can lead to an increased density of receptors. Evidence suggests that acute $(8,9,12)$ and chronic (32) treatment with anesthetics targeting NMDARs results in the upregulation of NMDA receptors as assessed by the levels of GluN2B subunit protein and mRNA. Since GluN2B-containing receptors are highly sensitive to isoflurane compared to GluN2Acontaining receptors (33), the finding that only GluN2B was upregulated in the hippocampus, whereas GluN2A was not altered, can be attributed to the high affinity of isoflurane for GluN2B-containing receptors, which allows for the specific upregulation of GluN2B expression in the hippocampus following acute isoflurane exposure.

Furthermore, we found that extrasynaptic GluN2B protein expression, but not synaptic GluN2B, increased significantly after $4 \mathrm{~h}$ of isoflurane exposure compared to non-isoflurane exposure, and treatment with the GluN2B antagonist Ro25-6981 was able to alleviate this impairment. Since the majority of GluN2A is incorporated into synapses, and GluN2B is mainly found extrasynaptically, these outcomes indicate that extrasynaptic GluN2B subunit upregulation may be involved in isoflurane-induced cognitive dysfunction.

GluN2B subunits are particularly important for plasticity, learning and memory, and their location in synaptic and extrasynaptic compartments play different roles $(6,14,16,17,34-36)$. Elevations in the synaptic GluN2B subunit levels and GluN2B-containing NMDARs are involved in spatial learning tasks and hippocampal long-term potentiation (LTP) enhancement $(36,37)$, whereas intrasynaptic GluN2B reduction is accompanied by cognitive impairment $(16,38)$. On the other hand, activating extrasynaptic GluN2B can facilitate hippocampal long-term depression (LTD) and inhibit LTP $(34,35)$. Therefore, increasing extrasynaptic GluN2B expression by isoflurane exposure may produce inhibitory effects on synaptic transmission and cause loss of cognitive function.

The molecular machinery regulating the sub-cellular localization of GluN2B subunits remains to be elucidated. Evidence suggests that anesthetics and aging can disturb 
these mechanisms. For example, it has been recognized that phosphorylation of GluN2B is important for the regulation of such processes (39), which can be influenced by aging and exposure to anesthetics (40-42). Increasing levels of CDK5 with age are capable of regulating the binding of Src to PSD-95, leading to dephosphorylation of tyrosine-1472 of GluN2B at synapses and its endocytosis, and reducing levels of synaptic GluN2B, but does not affect extrasynaptic GluN2B levels $(43,44)$. Therefore, isoflurane-induced upregulation of GluN2B, combined with increased CDK5 in aging rats, which curtails the synaptic location of GluN2B by dephosphorylation of tyrosine-1472, or by directly phosphorylating GluN2B at Serine-1116, decreasing its synaptic expression (45) may explain our result that intrasynaptic GluN2B expression showed no changes, while there was upregulation of total and extrasynaptic GluN2B. The schematic in Fig. 4 outlines the process. The animal model used by Rammes et al (8) was an adult model and may have had a relatively lower activity of CDK5 and lower GluN2B endocytosis compared to aged animals, and consequently, the upregulation of total GluN2B may lead to increased synaptic GluN2B and improved cognitive function.

Although previous findings have shown that systemic or intracerebral administration of NMDAR antagonists affects learning and memory performance on various tasks, including spatial learning and memory test in MWM (46-48), our findings showed that rats treated with Ro25-6981 alone have no changes in spatial learning and memory compared with the control animals. However, rats exposed to isoflurane and Ro25-6981 present improved cognitive function compared to the isoflurane group. Possible explanations include that intrasynaptic GluN2B-containing NMDA receptors are not sensitive to the small dose of Ro25-6981 used in our investigation under 'normal' physiological conditions, or the latter cannot pass through blood-brain barrier without isoflurane (2). A higher dose of Ro25-6981 may interfere with NMDARs function severely and lead to cognitive impairment $(8,48)$.

In conclusion, the present data suggest that isoflurane may induce reversible cognitive impairment, and isoflurane-induced sustained upregulation of extrasynaptic GluN2B, not synaptic GluN2B, after anesthesia, may be involved in this cognitive impairment. The precise mechanisms for general anesthetic-mediated modulation of the subcellular localization of GluN2B subunits and their role in cognitive function remain to be elucidated.

\section{Acknowledgements}

The present study was supported by grants from the National Natural Science Foundation of China (nos. 81371205, 81571036 and 81571044), and the National Basic Research Program of China (973 Program, no. 2012CB911004).

\section{References}

1. Li ZQ, Rong XY, Liu YJ, Ni C, Tian XS, Mo N, Chui DH and Guo XY: Activation of the canonical nuclear factor- $\kappa \mathrm{B}$ pathway is involved in isoflurane-induced hippocampal interleukin-1 $\beta$ elevation and the resultant cognitive deficits in aged rats. Biochem Biophys Res Commun 438: 628-634, 2013.
2. Cao Y, Ni C, Li Z, Li L, Liu Y, Wang C, Zhong Y, Cui D and Guo X: Isoflurane anesthesia results in reversible ultrastructure and occludin tight junction protein expression changes in hippocampal blood-brain barrier in aged rats. Neurosci Lett 587: 51-56, 2015.

3. Jevtovic-Todorovic V, Absalom AR, Blomgren K, Brambrink A, Crosby G, Culley DJ, Fiskum G, Giffard RG, Herold KF, Loepke AW, et al: Anaesthetic neurotoxicity and neuroplasticity: an expert group report and statement based on the BJA Salzburg Seminar. Br J Anaesth 111: 143-151, 2013.

4. Rappaport BA, Suresh S, Hertz S, Evers AS and Orser BA: Anesthetic neurotoxicity-clinical implications of animal models. N Engl J Med 372: 796-797, 2015.

5. Zurek AA, Yu J, Wang DS, Haffey SC, Bridgwater EM, PennaA, Lecker I, Lei G, Chang T, Salter EW, et al: Sustained increase in $\alpha 5 \mathrm{GABAA}$ receptor function impairs memory after anesthesia. J Clin Invest 124: 5437-5441, 2014.

6. Shipton OA and Paulsen O: GluN2A and GluN2B subunitcontaining NMDA receptors in hippocampal plasticity. Philos Trans R Soc Lond B Biol Sci 369: 20130163, 2013.

7. Lee YS and Silva AJ: The molecular and cellular biology of enhanced cognition. Nat Rev Neurosci 10: 126-40, 2009.

8. Rammes G, Starker LK, Haseneder R, Berkmann J, Plack A, Zieglgänsberger W, Ohl F, Kochs EF and Blobner M: Isoflurane anaesthesia reversibly improves cognitive function and long-term potentiation (LTP) via an up-regulation in NMDA receptor 2B subunit expression. Neuropharmacology 56: 626-636, 2009.

9. Mawhinney LJ, de Rivero Vaccari JP, Alonso OF, Jimenez CA, Furones C, Moreno WJ, Lewis MC, Dietrich WD and Bramlett HM: Isoflurane/nitrous oxide anesthesia induces increases in NMDA receptor subunit NR2B protein expression in the aged rat brain. Brain Res 1431: 23-34, 2012.

10. Chi H, Kawano T, Tamura T, Iwata H, Takahashi Y, Eguchi S, Yamazaki F, Kumagai N and Yokoyama M: Postoperative pain impairs subsequent performance on a spatial memory task via effects on N-methyl-D-aspartate receptor in aged rats. Life Sci 93: 986-993, 2013

11. Wang WY, Jia LJ, Luo Y, Zhang HH, Cai F, Mao H, Xu WC, Fang JB, Peng ZY, Ma ZW, et al: Location- and subunit-specific NMDA receptors determine the developmental sevoflurane neurotoxicity through ERK1/2 signaling. Mol Neurobiol 53: 216-230, 2016.

12. Liu J, Zhang X, Zhang W, Gu G and Wang P: Effects of sevoflurane on young male adult C57BL/6 mice spatial cognition. PLoS One 10: e0134217, 2015.

13. Ivanov A, Pellegrino C, Rama S, Dumalska I, Salyha Y, Ben-Ari Y and Medina I: Opposing role of synaptic and extrasynaptic NMDA receptors in regulation of the extracellular signal-regulated kinases (ERK) activity in cultured rat hippocampal neurons. J Physiol 572: 789-798, 2006.

14. Hardingham GE and Bading H: Synaptic versus extrasynaptic NMDA receptor signalling: Implications for neurodegenerative disorders. Nat Rev Neurosci 11:682-696, 2010.

15. Soria FN, Pérez-Samartín A, Martin A, Gona KB, Llop J, Szczupak B, Chara JC, Matute C and Domercq M: Extrasynaptic glutamate release through cystine/glutamate antiporter contributes to ischemic damage. JClin Invest 124: 3645-3655, 2014.

16. Zhang X, Xin X, Dong Y, Zhang Y, Yu B, Mao J and Xie Z: Surgical incision-induced nociception causes cognitive impairment and reduction in synaptic NMDA receptor $2 \mathrm{~B}$ in mice. J Neurosci 33: 17737-17748, 2013.

17. Goebel-Goody SM, Davies KD, Alvestad Linger RM, Freund RK and Browning MD: Phospho-regulation of synaptic and extrasynaptic N-methyl-d-aspartate receptors in adult hippocampal slices. Neuroscience 158: 1446-1459, 2009.

18. Hudson $\mathrm{AE}$ and Hemmings $\mathrm{HC}$ Jr: Are anaesthetics toxic to the brain? Br J Anaesth 107: 30-7, 2011.

19. Rappaport BA, Suresh S, Hertz S, Evers AS and Orser BA: Anesthetic neurotoxicity-clinical implications of animal models. N Engl J Med 372: 796-7, 2015.

20. Brambrink AM, Orfanakis A and Kirsch JR: Anesthetic neurotoxicity. Anesthesiol Clin 30: 207-228, 2012.

21. Moller JT, Cluitmans P, Rasmussen LS, Houx P, Rasmussen H, Canet J, Rabbitt P, Jolles J, Larsen K, Hanning CD, et al: Long-term postoperative cognitive dysfunction in the elderly ISPOCD1 study. ISPOCD investigators. International study of post-operative cognitive dysfunction. Lancet 351: 857-861, 1998.

22. Newman S, Stygall J, Hirani S, Shaefi S and Maze M: Postoperative cognitive dysfunction after noncardiac surgery: A systematic review. Anesthesiology 106: 572-590, 2007. 
23. Nadelson MR, Sanders RD and Avidan MS: Perioperative cognitive trajectory in adults. Br J Anaesth 112: 440-451, 2014.

24. Stratmann G, Sall JW, Bell JS, Alvi RS, May L, Ku B, Dowlatshahi M, Dai R, Bickler PE, Russell I, et al: Isoflurane does not affect brain cell death, hippocampal neurogenesis, or long-term neurocognitive outcome in aged rats. Anesthesiology 112: 305-315, 2010.

25. Liu H and Weng H: Up-regulation of Alzheimer's disease-associated proteins may cause enflurane anesthesia induced cognitive decline in aged rats. Neurol Sci 35: 185-189, 2014.

26. Selkoe DJ: Alzheimer's disease is a synaptic failure. Science 298: 789-791, 2002.

27. Arendt T: Synaptic degeneration in Alzheimer's disease. Acta Neuropathol 118: 167-179, 2009.

28. Querfurth HW and LaFerla FM: Alzheimer's disease. N Engl J Med 362: 329-344, 2010

29. Shipton OA, Leitz JR, Dworzak J, Acton CE, Tunbridge EM, Denk F, Dawson HN, Vitek MP, Wade-Martins R, Paulsen O, et al: Tau protein is required for amyloid \{beta\}-induced impairment of hippocampal long-term potentiation. J Neurosci 31: 1688-1692, 2011.

30. Pooler AM, Noble W and Hanger DP: A role for tau at the synapse in Alzheimer's disease pathogenesis. Neuropharmacology 76 Pt A: 1-8, 2014. doi: 10.1016/j.neuropharm.2013.09.018.

31. Parsons MP and Raymond LA: Extrasynaptic NMDA receptor involvement in central nervous system disorders. Neuron 82: 279-293, 2014.

32. Nagy J: The NR2B subtype of NMDA receptor: a potential target for the treatment of alcohol dependence. Curr Drug Targets CNS Neurol Disord 3: 169-179, 2004.

33. Ming Z, Griffith BL, Breese GR, Mueller RA and Criswell HE: Changes in the effect of isoflurane on N-methyl-D-aspartic acidgated currents in cultured cerebral cortical neurons with time in culture: evidence for subunit specificity. Anesthesiology 97 : 856-867, 2002

34. Liu DD, Yang Q and Li ST: Activation of extrasynaptic NMDA receptors induces LTD in rat hippocampal CA1 neurons. Brain Res Bull 93: 10-16, 2013.

35. Li S, Jin M, Koeglsperger T, Shepardson NE, Shankar GM and Selkoe DJ: Soluble A $\beta$ oligomers inhibit long-term potentiation through a mechanism involving excessive activation of extrasynaptic NR2B-containing NMDA receptors. J Neurosci 31: 6627-6638, 2011.

36. Tang YP, Shimizu E, Dube GR, Rampon C, Kerchner GA, Zhuo M, Liu G and Tsien JZ: Genetic enhancement of learning and memory in mice. Nature 401: 6369, 1999.

37. Hawasli AH, Benavides DR, Nguyen C, Kansy JW, Hayashi K, Chambon P, Greengard P, Powell CM, Cooper DC and Bibb JA: Cyclin-dependent kinase 5 governs learning and synaptic plasticity via control of NMDAR degradation. Nat Neurosci 10: 880-886, 2007.
38. Zhao X, Rosenke R, Kronemann D, Brim B, Das SR, Dunah AW and Magnusson KR: The effects of aging on $\mathrm{N}$-methyl-D-aspartate receptor subunits in the synaptic membrane and relationships to long-term spatial memory. Neuroscience 162: 933-945, 2009.

39. Chen BS and Roche KW: Regulation of NMDA receptors by phosphorylation. Neuropharmacology 53: 362, 2007.

40. Pehar M, Ko MH, Li M, Scrable H and Puglielli L: P44, the 'longevity-assurance' isoform of P53, regulates tau phosphorylation and is activated in an age-dependent fashion. Aging Cell 13: 449-456, 2014.

41. Song WJ, Son MY, Lee HW, Seo H, Kim JH and Chung SH: Enhancement of BACE1 activity by $\mathrm{p} 25 / \mathrm{Cdk} 5$-mediated phosphorylation in Alzheimer's disease. PLoS One 10: e0136950, 2015.

42. Wang WY, Luo Y, Jia LJ, Hu SF, Lou XK, Shen SL, Lu H, Zhang HH, Yang R, Wang H, et al: Inhibition of aberrant cyclin-dependent kinase 5 activity attenuates isoflurane neurotoxicity in the developing brain. Neuropharmacology 77: 90-99, 2014.

43. Zhang S, Edelmann L, Liu J, Crandall JE and Morabito MA: Cdk5 regulates the phosphorylation of tyrosine 1472 NR2B and the surface expression of NMDA receptors. J Neurosci 28: 415-424, 2008.

44. Sato S, Xu J, Okuyama S, Martinez LB, Walsh SM, Jacobsen MT, Swan RJ, Schlautman JD, Ciborowski P and Ikezu T: Spatial learning impairment, enhanced CDK5/p35 activity, and downregulation of NMDA receptor expression in transgenic mice expressing tau-tubulin kinase 1. J Neurosci 28: 14511-14521, 2008.

45. Plattner F, Hernández A, Kistler TM, Pozo K, Zhong P, Yuen EY, Tan C, Hawasli AH, Cooke SF, Nishi A, et al: Memory enhancement by targeting Cdk5 regulation of NR2B. Neuron 81: 1070-1083, 2014.

46. Howland JG and Cazakoff BN: Effects of acute stress and GluN2B-containing NMDA receptor antagonism on object and object-place recognition memory. Neurobiol Learn Mem 93: 261-267, 2010

47. Ge Y, Dong Z, Bagot RC, Howland JG, Phillips AG, Wong TP and Wang YT: Hippocampal long-term depression is required for the consolidation of spatial memory. Proc Natl Acad Sci USA 107: 16697-16702, 2010.

48. Mathur P, Graybeal C, Feyder M, Davis MI and Holmes A: Fear memory impairing effects of systemic treatment with the NMDA NR2B subunit antagonist, Ro 25-6981, in mice: attenuation with ageing. Pharmacol Biochem Behav 91: 453-460, 2009. 